\title{
CO Chemisorption at Metal Surfaces and Overlayers
}

\author{
Hammer, Bjørk; Morikawa, Y.; Nørskov, Jens Kehlet
}

Published in:

Physical Review Letters

Link to article, DOI:

10.1103/PhysRevLett.76.2141

Publication date:

1996

Document Version

Publisher's PDF, also known as Version of record

Link back to DTU Orbit

Citation (APA):

Hammer, B., Morikawa, Y., \& Nørskov, J. K. (1996). CO Chemisorption at Metal Surfaces and Overlayers. Physical Review Letters, 76(12), 2141-2144. https://doi.org/10.1103/PhysRevLett.76.2141

\section{General rights}

Copyright and moral rights for the publications made accessible in the public portal are retained by the authors and/or other copyright owners and it is a condition of accessing publications that users recognise and abide by the legal requirements associated with these rights.

- Users may download and print one copy of any publication from the public portal for the purpose of private study or research.

- You may not further distribute the material or use it for any profit-making activity or commercial gain

- You may freely distribute the URL identifying the publication in the public portal

If you believe that this document breaches copyright please contact us providing details, and we will remove access to the work immediately and investigate your claim 


\title{
CO Chemisorption at Metal Surfaces and Overlayers
}

\author{
B. Hammer, ${ }^{1,2}$ Y. Morikawa, ${ }^{2}$ and J. K. Nørskov ${ }^{1}$ \\ ${ }^{1}$ Center for Atomic-scale Materials Physics and Physics Department, Technical University of Denmark, DK-2800 Lyngby, Denmark \\ ${ }^{2}$ Joint Research Center for Atom Technology (JRCAT), 1-1-4 Higashi, Tsukuba, Ibaraki 305, Japan
}

(Received 31 August 1995)

A database of $a b$ initio calculations of the chemisorption energy of $\mathrm{CO}$ over $\mathrm{Ni}(111), \mathrm{Cu}(111)$, $\mathrm{Ru}(0001), \operatorname{Pd}(111), \operatorname{Ag}(111), \operatorname{Pt}(111), \mathrm{Au}(111), \mathrm{Cu}_{3} \mathrm{Pt}(111)$, and some metallic overlayer structures is presented. The trends can be reproduced with a simple model describing the interaction between the metal $d$ states and the $\mathrm{CO} 2 \pi^{*}$ and $5 \sigma$ states, renormalized by the metal $s p$ continuum. Our model rationalizes the results by Rodriguez and Goodman [Science 257, 897 (1992)] showing a strong correlation between the $\mathrm{CO}$ chemisorption energy and the surface core level shift.

PACS numbers: 73.20.At, 71.15.Mb, 73.61.At, 82.65.My

Over the past three decades the field of surface science has produced a series of accurate spectroscopical techniques that can provide detailed information about the electronic structure at surfaces [1]. It would be extremely useful if spectroscopic data could also be used directly to give information about the chemical activity of the surface. This would open up new possibilities in the future search for, e.g., more efficient catalysts. Recently, Rodriguez and Goodman [2] have established a spectacular correlation between spectroscopical data (surface core level shifts) and the chemisorption energy of $\mathrm{CO}$ on a series of metal surfaces and overlayers. If such an approach can be generalized, we would have a means of predicting the chemical activity of surfaces based on the surface electronic properties alone.

In the present Letter, we discuss the physics of $\mathrm{CO}$ adsorption over metal surfaces and overlayers by presenting an extensive $a b$ initio database of $\mathrm{CO}$ chemisorption energies calculated within density functional theory (DFT) using the generalized gradient approximation (GGA). We demonstrate that the trends in the database can be understood using a simple two-level model describing the coupling of the CO $5 \sigma$ and $2 \pi^{*}$ states to the metal $d$ valence states. One key surface parameter determining the strength of the bonding turns out to be the energy of the center of the metal $d$ band. This surface property can be obtained from spectroscopical methods either directly with photoemission (UPS) or indirectly through the surface core level shifts $[3,4]$. Using this, we demonstrate explicitly how our model of the CO chemisorption energy can account for all of the experimental data of Rodriguez and Goodman.

Before presenting the DFT-GGA database we first discuss our simple model of the trends in $\mathrm{CO}$ chemisorption energies. When an atom or a molecule is adsorbed on simple metal surfaces like $\mathrm{Na}, \mathrm{Mg}$, or $\mathrm{Al}$ without $d$ states, the electronic states of the adsorbate are broadened into resonances and shifted down in energy through the interaction with the broad continuum of metal $s p$ states $[5,6]$. This is illustrated in Fig. 1. While the adsorbate states may con- sist of closed shells the metallic $s p$ states have formed open bands, which enable energy gain through hybridization of adsorbate and metal electronic states. These metal surfaces, thereby, build up bonds to many adsorbates, including $\mathrm{CO}$ [7]. The surfaces of transition metals and noble metals also have open $s p$ bands and therefore also form bonds to adsorbates. However, the presence of the $d$ states in these metals enables a further bonding interaction between the metal $d$ states and the adsorbate related states (that are already renormalized through the interaction with the metal $s p$ states) [8-13] as illustrated in Fig. 1. It can easily be shown in a tight-binding framework that the total energy change caused by this interaction takes the form of a hybridization energy gain and an orthogonalization energy cost. In the limit of a small overlap $S$ between the adsorbate states and the metal $d$ states and of a small coupling matrix element $V$ compared to the energy separation,

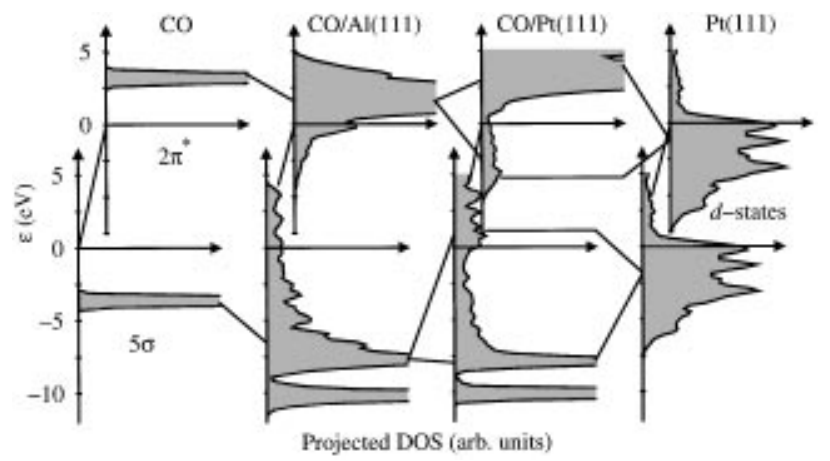

FIG. 1. The self-consistent electronic density of states (DOS) projected onto the $5 \sigma$ and $2 \pi^{*}$ orbitals of CO: in vacuum and over $\mathrm{Al}(111)$ and $\mathrm{Pt}(111)$ surfaces. Also shown is the DOS from the $d$ bands in the $\operatorname{Pt}(111)$ surface. The sharp states of $\mathrm{CO}$ in vacuum are seen to broaden into resonances and shift down in energy over the simple metal surface (mixing with the $4 \sigma$ state causes additional structure in the $5 \sigma$ resonance). Over the transition metal surfaces the $\mathrm{CO}$ resonances further hybridize with the metal $d$ states. This leads to shifts in the $5 \sigma$ and $2 \pi^{*}$ levels and to antibonding $5 \sigma-d$ states at the top of the $d$ bands and bonding $2 \pi^{*}-d$ states at the bottom. These states have low weight in the $5 \sigma$ and $2 \pi^{*}$ projections shown. 
$\Delta \epsilon$ between the two states, the hybridization energy gain becomes proportional to $V^{2} /|\Delta \epsilon|$ while the orthogonalization energy cost scales with $S V$ (i.e., roughly as $V^{2}$ ).

For CO, adsorption experiments [14] as well as many theoretical studies $[10,11,15,16]$ suggest that the filled $5 \sigma$ and the doubly degenerate, empty $2 \pi^{*}$ electronic states are the ones mainly responsible for the bonding to metal surfaces. We therefore write the following simple model expression for the $d$ contribution to the $\mathrm{CO}$ chemisorption energy over transition metal surfaces:

$$
\begin{aligned}
E_{d-\mathrm{hyb}} \simeq & -4\left[f \frac{V_{\pi}^{2}}{\epsilon_{2 \pi}-\epsilon_{d}}+f S_{\pi} V_{\pi}\right] \\
& -2\left[(1-f) \frac{V_{\sigma}^{2}}{\epsilon_{d}-\epsilon_{5 \sigma}}+(1+f) S_{\sigma} V_{\sigma}\right],
\end{aligned}
$$

where 2 is for spin, $f$ is the fractional filling of the $d$ bands, $\epsilon_{2 \pi}$ and $\epsilon_{5 \sigma}$ are the positions in energy of the (renormalized) adsorbate states, and $\epsilon_{d}$ is the center of the metal $d$ bands. $V$ and $S$ are labeled according to the symmetry of the orbitals they describe.

The fractional filling factors, the coupling matrix elements, and the overlaps in Eq. (1) we take to be dependent only on the atomic number of the metal atom to which the $\mathrm{CO}$ bonds, i.e., independent on the environment of this metal atom. The environment will manifest itself through the position of the center of the $d$ states on the metal atom in the surface before the $\mathrm{CO}$ adsorption. Values for the center, determined by DFT calculations, are included in Table I. We approximate $f$ with the idealized fractional filling factor $(v-1) / 10$ where $v$ is the valence of the metal atom. As we will be concerned with the variation of the $E_{d \text {-hyb }}$ from one metal to the next it suffices to estimate $V_{\pi}$ and $V_{\sigma}$ in an LMTO (linear muffin tin orbital) framework [17], where they factorize as products of terms dependent only on the adsorbate and the substrate properties, respectively. This means that the present coupling matrix elements must scale precisely as the LMTO based $V_{s d}$ used in Ref. [13] for a different adsorbate $\mathrm{H}_{2}$ interacting with the transition metal surfaces. Introducing $\alpha$ and $\beta$ as adjustable parameters common to all the metals, we write $V_{\pi}^{2} \simeq \beta V_{s d}^{2}$ and $S_{\pi} \simeq-\alpha V_{\pi}$. From the DFT orbitals of CO and the various metals we find that $S_{\sigma} / S_{\pi} \simeq 1.3$ is a good approximation and we therefore write $V_{\sigma}^{2} \simeq(1.3)^{2} \beta V_{s d}^{2}$ and $S_{\sigma} \simeq-\alpha V_{\sigma}$.

As the transition metal surfaces considered have very similar half filled $s$ bands, the renormalization of the $\mathrm{CO}$ states by the delocalized metal states will be very alike. Guided by the results of DFT calculations for $\mathrm{CO}$ adsorption on $\mathrm{Al}(111)$ (Fig. 1) and on the transition metal surfaces with small coupling matrix elements, we use +2.5 and $-7 \mathrm{eV}$ (with respect to the Fermi level) for the renormalized $\epsilon_{2 \pi}$ and $\epsilon_{5 \sigma}$ positions, respectively.

In Fig. 2 we present the scaling of the chemisorption energy for $\mathrm{CO}$ within the model as compared to the set of $a b$ initio DFT calculations given in Table I. Adjusting only $\alpha$ and $\beta$ in the model, we obtain the excellent correlation in Fig. 2 . $\alpha$ and $\beta$ values of $0.063 \mathrm{eV}^{-1}$
TABLE I. Parameters and results for $\mathrm{CO}$ chemisorption atop a metal atom (first column) in metal surfaces and overlayers [second column: $M_{1} / M_{2}$ means a monolayer of $M_{1}$ on a $M_{2}$ substrate. "Ni@ $\mathrm{Cu}(111)$ " refers to a $\mathrm{Cu}(111)$ substrate with every fourth surface $\mathrm{Cu}$ substituted by a Ni]. The center $\epsilon_{d}$ of the local $d$ bands at the metal atom measured relative to the Fermi level and the fractional filling $f$ of the these bands. The coupling matrix element $V_{s d}^{2}$ (normalized to 1.0 for $\mathrm{Cu}$ ) and the chemisorption energy $E_{\mathrm{chem}}$ from the DFTGGA calculations. The last column gives the experimental chemisorption energies for $\mathrm{CO}$ on $\mathrm{Ni}(111)$ [27], $\mathrm{Cu}(111)$ [28], $\mathrm{Ru}(0001)$ [29], $\operatorname{Pd}(111)$ [30], $\operatorname{Ag}(111)$ [31], $\operatorname{Pt}(111)$ [32], and $\mathrm{Al}(111)$ [7]. All energies are in $\mathrm{eV}$.

\begin{tabular}{llccccc}
\hline \hline Atop & \multicolumn{1}{c}{ Surface } & $\epsilon_{d}$ & $f$ & $V_{s d}^{2}$ & $E_{\text {chem }}$ & $E_{\text {exp }}$ \\
\hline $\mathrm{Ni}$ & $\mathrm{Ni}(111)$ & -1.48 & 0.9 & 1.16 & -1.36 & -1.26 \\
$\mathrm{Ni}$ & $\mathrm{Ni} / \mathrm{Ru}(1000)$ & -1.27 & 0.9 & 1.16 & -1.51 & \\
$\mathrm{Ni}$ & $\mathrm{Ni} @ \mathrm{Cu}(111)$ & -1.18 & 0.9 & 1.16 & -1.56 & \\
$\mathrm{Cu}$ & $\mathrm{Cu}(111)$ & -2.67 & 1.0 & 1.00 & -0.62 & -0.52 \\
$\mathrm{Cu}$ & $\mathrm{Cu} / \mathrm{Pt}(111)$ & -1.88 & 1.0 & 1.00 & -0.94 & \\
$\mathrm{Cu}$ & $\mathrm{Ni} @ \mathrm{Cu}(111)$ & -2.56 & 1.0 & 1.00 & -0.61 & \\
$\mathrm{Cu}$ & $\mathrm{Cu}{ }_{3} \mathrm{Pt}(111)$ & -2.35 & 1.0 & 1.00 & -0.53 & \\
$\mathrm{Ru}$ & $\mathrm{Ru}(0001)$ & -1.41 & 0.7 & 3.87 & -1.80 & -1.66 \\
$\mathrm{Pd}$ & $\mathrm{Pd}(111)$ & -2.16 & 0.9 & 2.78 & -1.30 & -1.47 \\
$\mathrm{Pd}$ & $\mathrm{Pd} / \mathrm{Ru}(0001)$ & -2.86 & 0.9 & 2.78 & -0.98 & \\
$\mathrm{Ag}$ & $\mathrm{Ag}(111)$ & -4.28 & 1.0 & 2.26 & 0.09 & -0.28 \\
$\mathrm{Pt}$ & $\mathrm{Pt}(111)$ & -2.75 & 0.9 & 3.90 & -1.45 & -1.50 \\
$\mathrm{Pt}$ & $\mathrm{Cu}{ }_{3} \mathrm{Pt}(111)$ & -2.55 & 0.9 & 3.90 & -1.51 & \\
$\mathrm{Au}$ & $\mathrm{Au}(111)$ & -3.91 & 1.0 & 3.35 & -0.04 & \\
$\mathrm{Al}$ & $\mathrm{Al}(111)$ & & & & -0.49 & -0.21 \\
\hline \hline
\end{tabular}

and $1.5 \mathrm{eV}^{2}$, respectively, have been used, both of which are of the right order of magnitude compared to DFT estimates [17] of $0.09 \mathrm{eV}^{-1}$ and $2 \mathrm{eV}^{2}$. We note that in Fig. 2 the slope of the least square fitted curve guiding the eye is close to one, which means that the adsorbate-metal $d$ interactions described by our model can account for the main trends in $\mathrm{CO}$ bonding from one surface to the next. The curve is offset by $\sim 0.5 \mathrm{eV}$ on the vertical axis for $E_{d \text {-hyb }}=0$, which fits with CO forming a bond of this strength on simple metal surfaces (see Table I). Outside the scale in Fig. 2 is the result for $\mathrm{CO} / \mathrm{Ru}(0001)$. Here the simple model [Eq. (1)] estimates a $d$ contribution of $-2.08 \mathrm{eV}$ to the chemisorption energy. Both the model and the full calculation thus show that the CO-Ru bond is very strong. However, in this case (as for most of the metals to the left in the transition metal series) where $\epsilon_{2 \pi}-\epsilon_{d}$ is small and the $d$ band width is large, the neglect of the latter in the two level model of Eq. (1) leads to a largely overestimated $E_{d \text {-hyb }}$.

As an important property of the model, we further note that it captures the shifts in the CO chemisorption energy from the single crystal surfaces to the overlayer structures. This is apparent in Fig. 2 as seen by the dashed lines. For $\mathrm{Cu}_{3} \mathrm{Pt}$, which is a very stable covalently bonded alloy, the $\mathrm{Cu}$ sites become less reactive than predicted by the model, while the Pt sites behave roughly as expected. As the model only describes the coupling of the metal $d$ states to the renormalized $\mathrm{CO} 2 \pi^{*}$ and $5 \sigma$ states we conclude 


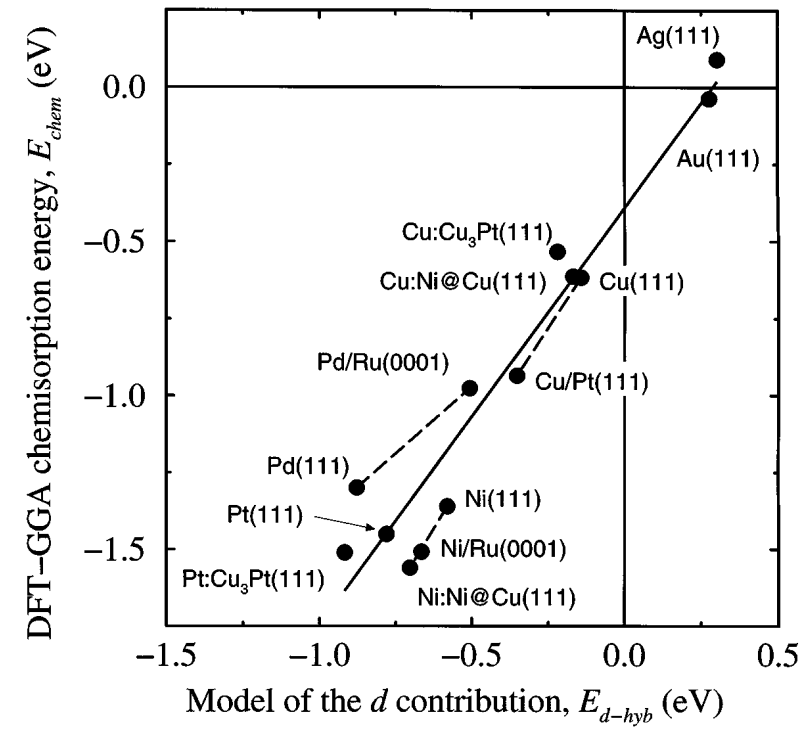

FIG. 2. Comparison of the model and the full DFT-GGA chemisorption energies for a number of metal systems.

that it is this interaction which is responsible not only for the gross trends in $\mathrm{CO}$ chemisorption energies over the wide range of the late transition metal surfaces considered but also for the details for metallic overlayers and alloy surfaces. We return to this below.

The ab initio DFT calculations [18] are performed using the local density approximation (LDA) [19] for finding self-consistent charge densities and densities of states (DOS) while using for the exchange-correlation energy in all reported total energy differences the GGA [20]. A quarter monolayer of $\mathrm{CO}$ is adsorbed on one side of slabs having six fcc (111)—for Ru: hcp (0001) layers of metal atoms. Ionic cores are described with pseudopotentials [21]. The Kohn-Sham equations are solved in a basis of plane waves of kinetic energy up to $40 \mathrm{Ry}$ (for $\mathrm{Ni}$ and $\mathrm{Cu}$ : up to $50 \mathrm{Ry}$ ) at 6 and $15 \mathrm{k}$ points in the $C_{3 v}$ and $C_{2 v}$ irreducible Brillouin zones, respectively.

For the present purposes, we choose to consider the DFT-GGA calculations as a computer experiment of $\mathrm{CO}$ adsorption on a number of metal surfaces with all ionic degrees of freedom kept fixed. Hereby we can concentrate on the ability of the model [Eq. (1)] to capture the trends caused by the electronic factors of the CO-metal bonding. Substrate relaxations are therefore not considered, but rather the truncated bulk geometries are used [22]. Further, for all surfaces, $\mathrm{CO}$ is put at the top position with a fixed metal-carbon distance of $1.94 \AA$ and a $\mathrm{CO}$ bond length of $1.14 \AA$ as reported from calculations for $\mathrm{CO} / \mathrm{Pd}(110)$ [23]. The use of fixed $\mathrm{CO}$ coordinates is actually a good approximation. For $\mathrm{CO}$ on $\mathrm{Pt}(111)$ we find that the relaxed values are $r_{\mathrm{MC}}=1.88 \AA$ and $r_{\mathrm{CO}}=1.15 \AA$ and that this relaxation influences $E_{\text {chem }}$ by less than $0.05 \mathrm{eV}$. That the DFT-GGA is capable of describing the $\mathrm{CO}$ itself and the CO-metal interaction well is suggested both by previous calculations for $\mathrm{Pd}$ and $\mathrm{Cu}$ surfaces [23,24] and by our calculations: For $\mathrm{CO}$ in vacuum we get a bond strength of $10.88 \mathrm{eV}$ and a vibrational frequency of $2162 \mathrm{~cm}^{-1}$ both of which compare well with the experimental values of $11.09 \mathrm{eV}$ and $2169.8 \mathrm{~cm}^{-1}$ [25]. For $\mathrm{CO}$ adsorbed atop $\mathrm{Pt}(111)$ we calculate a downshift of the $\mathrm{CO}$ stretch frequency to $2120 \mathrm{~cm}^{-1}$ in agreement with an experimentally observed downshift to $2104 \mathrm{~cm}^{-1}$ [26]. Finally, considering the use of fixed site and coordinates, the chemisorption energies in our database agree well overall with the experimentally determined heats of $\mathrm{CO}$ adsorption included in Table I. In particular, for $\operatorname{Ag}(111)$ and $\mathrm{Au}(111)$ where the orthogonalization terms dominate we expect an outward relaxation of the $\mathrm{CO}$ to influence the chemisorption energies bringing theory and experiment in better agreement.

Our present model of the $\mathrm{CO}$ bonding is in complete agreement with the theoretical interpretations developed by Blyholder [15], Bagus [11], and others. The language of electron donation from the $\mathrm{CO} 5 \sigma$ to the metal and backdonation from the metal to the $\mathrm{CO} 2 \pi^{*}$ describes the concerted action of the coupling of the $\mathrm{CO}$ levels to the metal $s p$ states and the $d$ states. With the present division of the donation and backdonation into separate metal $s p$ and $d$ steps which follows the reasoning of Bagus and Pacchioni [11] we obtain a simple picture and a quantitative model of the electronic reason for the trends in the $\mathrm{CO}$ chemisorption energies over metal surfaces and overlayers.

We now return to the experimental observation by Rodriguez and Goodman of a strong correlation between the surface core level shift of different overlayers and the CO chemisorption energy [2]. Our analysis goes in two steps. First, we build on the extensive theoretical insight into the origin of the surface core level shifts by Weinert and Watson (WW) [3] and Hennig, GandugliaPirovano, and Scheffler (HGS) [4]. WW show that the variation in surface core level shifts for metal overlayers is accompanied by a similar shift in the center of gravity of the $d$ bands-at least towards the right in the transition metal series, while charge transfer effects are inadequate for explaining the shifts. The latter is confirmed by HGS, who also show that the trends in variations in the surface core level shifts for different overlayers are given by the initial state shift, that is, by the changes in the electronic structure of the unperturbed surface. From this we conclude that we can view the variation in the surface core level shifts as a measure of the variation in the $d$ band center.

The second step in our analysis is then to use our model [Eq. (1)] to establish the relationship between variations in the $d$ band center and the chemisorption energy. We note that in Eq. (1) the hybridization energy term related to the $2 \pi^{*}$ dominates the expression. It therefore also dominates the differential change in $E_{d \text {-hyb }}$ for a change $\delta \epsilon_{d}$ in the position of the $d$-band center, which may be caused by changes in the surroundings of the metal atom at which the $\mathrm{CO}$ bonds. We have 


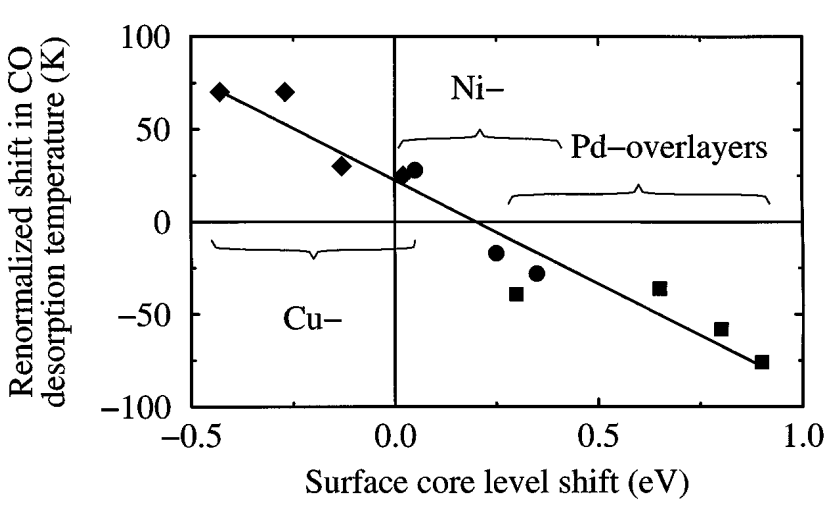

FIG. 3. The experimental data of Rodriguez and Goodman [2] for $\mathrm{CO}$ adsorption on $\mathrm{Ni}, \mathrm{Cu}$, and $\mathrm{Pd}$ overlayers plotted as the renormalized TPD peak shifts $(\Delta T / \gamma)$ vs the measured shifts in surface core level positions. The reference systems for the $\mathrm{CO}$ TPD peaks and the surface core level positions for the overlayer structures are the respective single crystal surfaces.

$\delta E_{d \text {-hyb }} \simeq\left[-4 f V_{\pi}^{2} /\left(\epsilon_{2 \pi}-\epsilon_{d}\right)^{2}\right] \delta \epsilon_{d}$. This suggests that different metal overlayers share the same direct proportionality relation between $\delta \epsilon_{d}$ and $\delta E_{d \text {-hyb }} / \gamma$, where $\gamma$ is proportional to $f V_{s p}^{2} /\left(\epsilon_{2 \pi}-\epsilon_{d}\right)^{2}$, which only depends on the overlayer metal and not on the substrate. For $\mathrm{Cu}$, $\mathrm{Ni}$, and Pd overlayers considered by Rodriguez and Goodman [2] $\gamma$ has values of $1,1.76$, and 3.08 relative to $\mathrm{Cu}$ (cf. Table I). In Fig. 3 we examine if this analysis can be used on the original experimental data [2]. The figure strongly supports our thesis. Identifying the shift in the $d$-band center with (minus) the core level shifts and assuming the $\mathrm{CO}$ chemisorption energy shift proportional to (minus) the temperature-programmed desorption (TPD) peak shift $(\Delta T / \gamma)$, a linear relation between the renormalized TPD peak shift and the core level shift is expected. Such a relationship is clearly present in Fig. 3, however, slightly offset from the origin. The offset might reflect a systematic offset in the core level shifts compared to the shifts of $d$-band centers.

The present work was in part financed by the Danish Research Councils through the Center for Surface Reactivity and Grant No. 9501775. Center for Atomic-scale Materials Physics is sponsored by the Danish National Research Foundation. JRCAT is supported by the New Energy and Industrial Technology Development Organization (NEDO) of Japan.

[1] D.P. Woodruff and T. A. Delchar, Modern Techniques of Surface Science (Cambridge University Press, Cambridge, 1986).

[2] J. A. Rodriguez and D. W. Goodman, Science 257, 897 (1992).

[3] M. Weinert and R.E. Watson, Phys. Rev. B 51, 17168 (1995).

[4] D. Hennig, M. V. Ganduglia-Pirovano, and M. Scheffler, Phys. Rev. B (to be published).

[5] D. M. Newns, Phys. Rev. 178, 1123 (1969).
[6] J. K. Nørskov, Rep. Prog. Phys. 53, 1253 (1990).

[7] T.-C. Chiang, G. Kaindl, and D.E. Eastman, Solid State Commun. 36, 25 (1980).

[8] B. I. Lundqvist, O. Gunnarsson, H. Hjelmberg, and J. K. Nørskov, Surf. Sci. 89, 196 (1979).

[9] S. Holloway, B.I. Lundqvist, and J. K. Nørskov, in Proceedings of the International Congress on Catalysis, Berlin, 1984 (Verlag Chemie, Weinheim, Germany, 1984), Vol. 4, pp. 85-95.

[10] R. Hoffmann, Rev. Mod. Phys. 60, 601 (1988).

[11] P. S. Bagus and G. Pacchioni, Surf. Sci. 278, 427 (1992).

[12] B. Hammer and M. Scheffler, Phys. Rev. Lett. 74, 3487 (1995).

[13] B. Hammer and J. K. Nørskov, Nature (London) 376, 238 (1995); Surf. Sci. 343, 211 (1995).

[14] J.C. Campuzano, in The Chemical Physics of Solid Surfaces and Heterogeneous Catalysis, edited by D. A. King and D.P. Woodruff (Elsevier, New York, 1990), Vol. 3A, p. 389.

[15] G. Blyholder, J. Phys. Chem. 68, 2772 (1964).

[16] M. A. van Daelen, Y.S. Li, J. M. Newsam, and R. A. van Santen, Chem. Phys. Lett. 226, 100 (1994).

[17] O. K. Andersen, O. Jepsen, and D. Glötzel, in Highlights of Condensed Matter Theory, Proceedings of the International School of Physics "Enrico Fermi," Course LXXXIX (North-Holland, Amsterdam, 1985), p. 59; J. K. Nørskov, J. Chem. Phys. 90, 7461 (1989).

[18] M. C. Payne, M. P. Teter, D. C. Allan, T.A. Arias, and J. D. Joannopoulos, Rev. Mod. Phys. 64, 1045 (1992).

[19] D. M. Ceperley and B. J. Alder, Phys. Rev. Lett. 45, 566 (1980); J. P. Perdew and A. Zunger, Phys. Rev. B 23, 5048 (1981).

[20] J. P. Perdew, J. A. Chevary, S. H. Vosko, K. A. Jackson, M. R. Pederson, D. J. Singh, and C. Fiolhais, Phys. Rev. B 46, 6671 (1992).

[21] G. B. Bachelet, D. R. Hamann, and M. Schlüter, Phys. Rev. B 26, 4199 (1982); N. Troullier and J. L. Martins, ibid. 43, 1993 (1991).

[22] When allowing for relaxations of the uppermost two layers of the clean $\mathrm{Cu} / \mathrm{Pt}(111)$ surface the DFT-GGA values for the $\mathrm{CO}$ chemisorption change by less than $0.01 \mathrm{eV}$.

[23] P. Hu, D. A. King, S. Crampin, M.-H. Lee, and M.C. Payne, Chem. Phys. Lett. 230, 501 (1994).

[24] P. H. T. Philipsen, G. te Velde, and E. J. Baerends, Chem. Phys. Lett. 226, 583 (1994).

[25] K.P. Huber and G. Herzberg, Constants of Diatomic Molecules, Molecular Spectra and Molecular Structure Vol. IV (Van Nostrand Reinhold, New York, 1979).

[26] E. Schweizer, B. N. J. Persson, M. Tüshaus, D. Hoge, and A. M. Bradshaw, Surf. Sci. 213, 49 (1989).

[27] J. B. Miller, H. R. Siddiqui, S. M. Gates, J. N. Russell, Jr., and J. T. Yates, Jr., J. Chem. Phys. 87, 6725 (1987).

[28] P. Hollins and J. Pritchard, Surf. Sci. 89, 486 (1979).

[29] H. Pfnür, P. Feulner, H. A. Engelhardt, and D. Menzel, Chem. Phys. Lett. 59, 481 (1978).

[30] H. Conrad, G. Ertl, J. Koch, and E. E. Latta, Surf. Sci. 43, 462 (1974).

[31] G. McElhiney, H. Papp, and J. Pritchard, Surf. Sci. 54, 617 (1976).

[32] H. Steininger, S. Lehwald, and H. Ibach, Surf. Sci. 123, 264 (1982). 